\title{
Réaction convergente du volume marchand 10 ans après l'éclaircie d'une sapinière très dense
}

\author{
par Martin Barrette ${ }^{1, *}$ et Stéphane Tremblay ${ }^{1}$
}

\begin{abstract}
RÉSUMÉ
Lautoéclaircie joue un rôle important dans le développement des peuplements forestiers. Ce processus écologique ne sopère pas efficacement à l'intérieur de certaines sapinières boréales très denses. Dans ces sapinières, la croissance des arbres a ralenti, ce qui a diminué les services écosystémiques rendus par le peuplement, tels que la production de matière ligneuse. Les principes sylvicoles de léclaircie par le bas se basent sur le processus écologique d’autoéclaircie. Ainsi, lobjectif de cette étude était de vérifier si l’application de ce traitement pouvait remplacer lautoéclaircie et rétablir la croissance dans une sapinière très dense ayant atteint lâge de la maturité commerciale. Dans un cadre expérimental, nous avons suivi lévolution de cette sapinière soumise à trois intensités déclaircie par le bas. Dix ans après l'intervention, les placettes fortement éclaircies avaient retrouvé un volume marchand semblable à celles non traitées. Ce volume était distribué sur $43 \%$ moins d'arbres, et le diamètre quadratique moyen a crû deux fois plus rapidement chez les 1000 plus gros d’entre eux. Il s'agit d'un des premiers cas recensés de réaction convergente du volume marchand après éclaircie. En définitive, nous avons démontré que léclaircie par le bas peut remplacer lautoéclaircie et rétablir la croissance dans des sapinières très denses.
\end{abstract}

Mots-clés: sapinières; autoéclaircie; éclaircie par le bas; sylviculture; réaction convergente de la production.

\section{ABSTRACT}

Self-thinning plays an important role in the development of forest stands. This ecological process does not operate effectively in certain very dense boreal balsam fir stands. In these stands, tree growth has slowed down, reducing ecosystem services such as timber production. Silvicultural principles of thinning from below are based on the self-thinning process. Hence, the objective of this study was to ascertain whether this treatment could replace the self-thinning process and restore growth in a very dense balsam fir stand that had reached the age of commercial maturity. We followed stand evolution after an experimental thinning from below at three different intensities. After 10 years, the heavily thinned plots recovered a level of merchantable volume similar to that of control plots. This volume was distributed on $43 \%$ fewer trees, and the mean quadratic diameter grew twice as rapidly for the 1000 largest of these trees. This is one of the first studies to document a convergent reaction of merchantable volume after thinning. Ultimately, we demonstrated that thinning from below can replace self-thinning and restore growth in very dense fir stands.

Keywords: balsam fir stands; self-thinning; thinning from below; silviculture; convergent reaction of production.

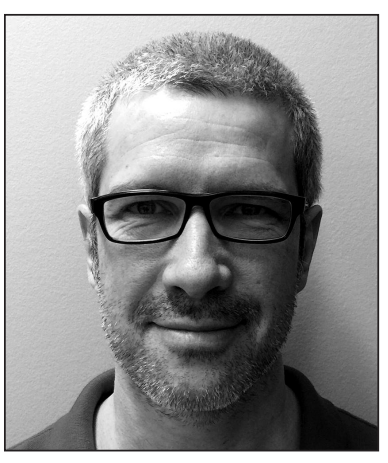

Martin Barrette

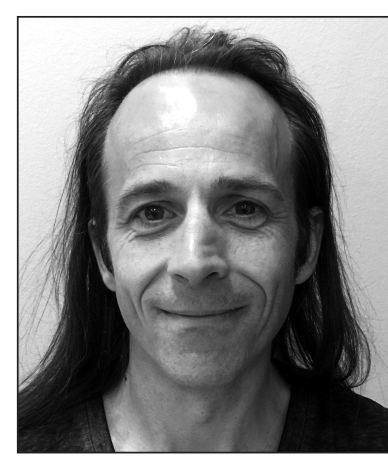

Stéphane Tremblay

\section{Introduction}

Lautoéclaircie joue un rôle important dans le développement des sapinières denses (McCarthy et Weetman 2007a). Ce processus écologique réduit la compétition reliée à la densité en causant la mort d'une portion des arbres. Plus de ressources et d'espace deviennent alors disponibles, ce qui favorise la croissance des survivants et le passage du peuplement vers la maturité (Smith et al. 1997). Le dysfonctionnement de l'autoéclaircie pourrait donc engendrer un ralentissement de croissance des arbres et une diminution des services écosystémiques rendus par le peuplement, tels que la production de matière ligneuse.

\footnotetext{
${ }^{1}$ Ministère des Forêts, de la Faune et des Parcs, Direction de la recherche forestière, Québec, QC

*Auteur pour correspondance. 2700, rue Einstein, Québec QC G1P 3W8, Canada courriel: martin.barrette@mffp.gouv.qc.ca
} 


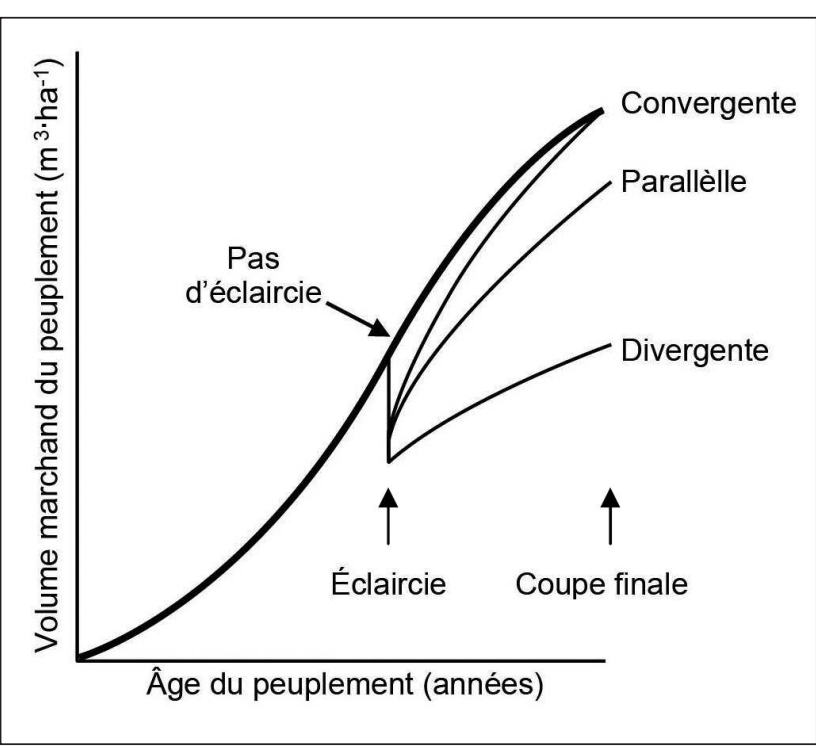

Fig. 1. Courbes conceptuelles de production de matière ligneuse de peuplements forestiers après éclaircie ladaptées de McKinnon et al. (2006) avec la permission du Ontario Ministry of Natural Resources; Copyright : Queen's Printer for Ontario, 2006).

Lautoéclaircie ne s'opère pas efficacement à l'intérieur de certaines sapinières boréales très denses. La croissance des arbres ralentit, de sorte que les caractéristiques de ces sapinières ne correspondent pas aux caractéristiques de sapinières se développant normalement (Raulier et al. 2003). Notamment, bien que les sapinières très denses aient atteint lâge de la maturité commerciale, leur volume est réparti sur un très grand nombre d'arbres de petite taille, au faible intérêt commercial (Raulier et al. 2003). Ce sont probablement les caractéristiques du sapin baumier (Abies balsamea [L.] Mill.) qui lont prédisposé à former des peuplements très denses. Tout d’abord, les sapinières matures peuvent produire une banque de semis très denses (Côté et Bélanger 1991, Morin et Laprise 1997). Ensuite, la grande tolérance à lombre du sapin baumier peut lui permettre de survivre à la compétition reliée à la densité pendant une période prolongée, sans qu'il perde sa capacité à réagir favorablement à louverture du couvert (McCarthy et Weetman 2007b, Barrette et al. 2010). Ainsi, si aucune perturbation ne vient éclaircir le peuplement issu d'une telle banque de semis, celui-ci pourrait maintenir une forte densité suffisamment longtemps pour que la compétition ralentisse la croissance.

Dans les sapinières où l'autoéclaircie ne s'opère pas efficacement, un traitement déclaircie par le bas pourrait rétablir la croissance, puisque les principes sylvicoles de ce traitement sont basés sur le processus écologique d'autoéclaircie (Franklin et al. 2007). En effet, léclaircie par le bas vise à diminuer la compétition reliée à la densité par la récolte des arbres les plus susceptibles de mourir (Smith et al. 1997). Ce traitement a donc pour effet de redistribuer les ressources et l'espace de croissance plus rapidement aux autres arbres, ce qui peut accélérer l'expansion de leur houppier et leur croissance en diamètre (McCarthy et Weetman 2007a, Nyland 2007). Lobjectif de cette étude est de vérifier expérimentalement si une éclaircie par le bas peut remplacer l'autoéclaircie et rétablir la croissance dans une sapinière ayant atteint la maturité commerciale. En raison des caractéristiques du sapin baumier, nous posons l'hypothèse que léclaircie rétablira la croissance des arbres augmentant ainsi la productivité de la sapinière. La production de matière ligneuse de la sapinière éclaircie convergera alors vers la production de la sapinière non éclaircie (Fig. 1; McKinnon et al. 2006).

\section{Matériel et méthodes \\ Aire d'étude}

Laire détude se situe dans la réserve faunique des Laurentides (Québec), dans le sous-domaine bioclimatique de la sapinière à bouleau blanc (Betula papyrifera Marsh.) de l'Est (Fig. 2; Saucier et al. 2009). La température moyenne annuelle est de $0,3{ }^{\circ} \mathrm{C}$ et les précipitations annuelles totales sont de $1527 \mathrm{~mm}$ (Environnement Canada 1993). Dans cette forêt boréale humide, où les feux sont peu fréquents, un régime cyclique dépidémies de tordeuse des bourgeons de lépinette (Choristoneura fumiferana (Clem.), TBE) favorise la prédominance du sapin baumier. Les sapinières appartenant à différentes classes d'âges s'entremêlent dans le paysage pour former une mosaïque hétérogène (Leblanc et Bélanger 2000). Le peuplement étudié a été sélectionné en fonction de sa représentati-

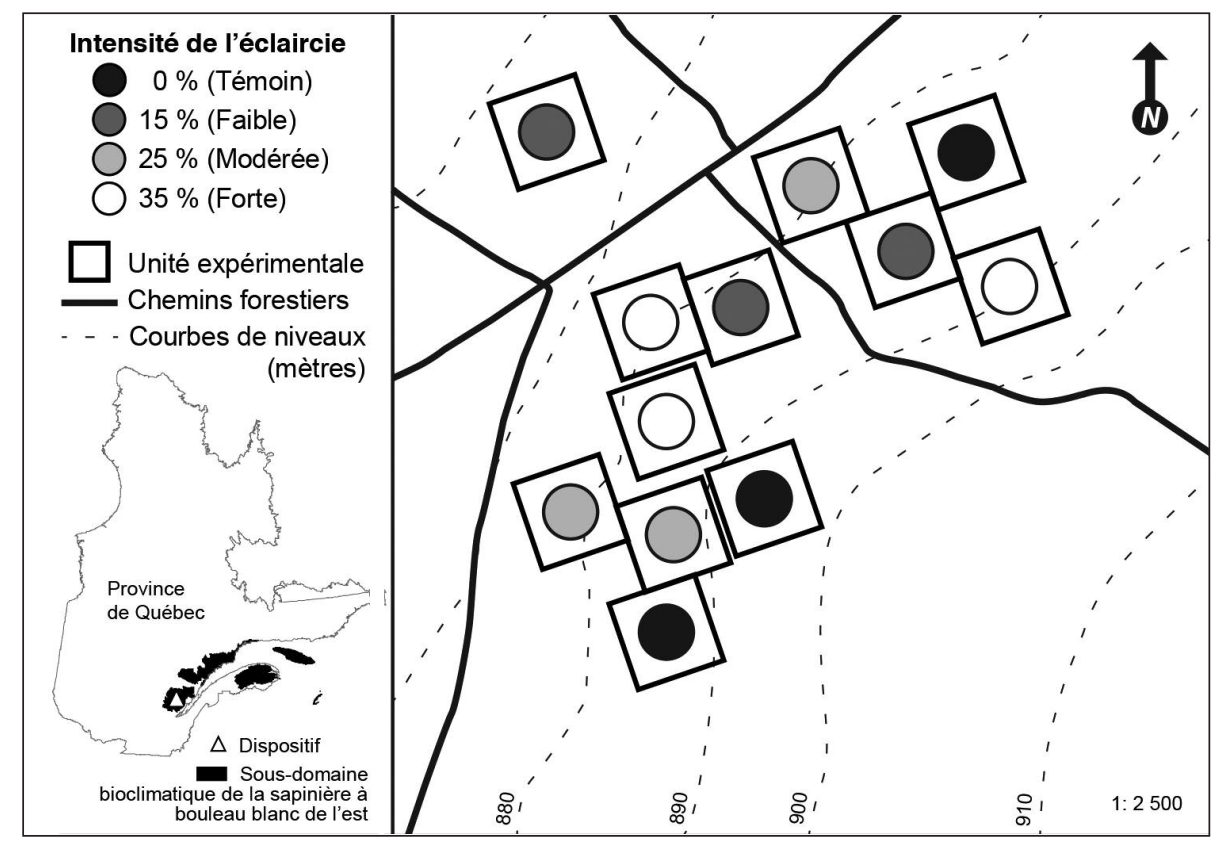

Fig. 2. Schéma du dispositif expérimental. Les carrés représentent les unités expérimentales (2500 $\mathrm{m}^{2}$ ) soumises à l'un des traitements d'éclaircie. Les cercles représentent les placettes principales $\left(400 \mathrm{~m}^{2}\right.$ ) utilisées pour les mesures. 
vité des sapinières très denses et de son accessibilité. Il est issu de régénération naturelle libérée par une coupe totale réalisée durant les années 1930. La station se trouve à une altitude de 860 à $900 \mathrm{~m}$, sur un till régional indifférencié avec un drainage mésique.

\section{Dispositif expérimental}

Le dispositif est composé de 12 unités expérimentales de 2500 m² $^{2} 50$ x 50 m; Fig. 2). En 1997, des intensités déclaircie de $0 \%$ (témoin), de $15 \%$ (faible), de $25 \%$ (modérée) et de $35 \%$ (forte) de la surface terrière marchande ont été attribuées aléatoirement aux unités expérimentales. Léclaircie par le bas a été réalisée uniformément sur l'ensemble des unités expérimentales, en ciblant les petits arbres ayant un diamètre à hauteur de poitrine $(\mathrm{DHP}) \geq 6,1 \mathrm{~cm}$. Nous qualifions cette éclaircie d'intermédiaire parce quelle a eu lieu dans un peuplement ayant atteint la maturité commerciale, mais ce ne sont pas seulement des arbres de taille marchande qui ont été coupés. Labattage s'est fait manuellement, et le dispositif est exempt de sentiers de débardage.

Une placette principale de $11,28 \mathrm{~m}$ de rayon $\left(400 \mathrm{~m}^{2}\right)$ ainsi qu'une placette secondaire concentrique de 5,64 $\mathrm{m}$ de rayon $\left(100 \mathrm{~m}^{2}\right)$ ont été placées au centre de chaque unité expérimentale. Avant l'éclaircie, nous avons mesuré le DHP (classes de $2 \mathrm{~cm}$ ) des arbres (DHP $\geq 1,1 \mathrm{~cm}$ ) de toutes les espèces dans les placettes principales. Lévolution des caractéristiques dendrométriques a ensuite été suivie 0,5 et 10 ans après l'éclaircie, soit en 1997, 2002 et 2007. Les arbres marchands $(\mathrm{DHP} \geq 9,1 \mathrm{~cm})$ de la placette principale ont été numérotés, et leur DHP a été mesuré avec un ruban circonférentiel ( $\mathrm{mm})$. Les gaules $(1,1 \mathrm{~cm} \leq \mathrm{DHP}<9,1 \mathrm{~cm})$ ont été dénombrées par classe de DHP $(2 \mathrm{~cm})$ dans les placettes secondaires. L'état (mort ou vivant) et l'espèce de tous les arbres ont aussi été notés. Cinq arbres échantillons de taille marchande ont été sélectionnés parmi les arbres représentatifs des étages dominant et codominant. Leur hauteur totale, la hauteur de la base de leur houppier vivant et leur âge à $1 \mathrm{~m}$ ont été mesurés sur le terrain. Au printemps 2009, des carottes ont été prélevées à $1 \mathrm{~m}$ sur les arbres échantillons avec une sonde de Pressler. Ces carottes ont permis l'analyse fine, par dendrochronologie, de l'ampleur et de la durée du ralentissement de croissance ainsi que de l'effet de l'éclaircie sur la croissance radiale des arbres. En laboratoire, les carottes de bois ont été séchées, collées sur des baguettes de bois, sablées et numérisées avec un scanneur. La largeur des cernes annuels a été mesurée à l'aide du logiciel WinDendro $^{\mathrm{MD}}$ (Version 6.01, Regent Instruments Inc., Quebec, Canada). Enfin, la datation des cernes a été vérifiée avec le logiciel COFECHA (Holmes 1983).

\section{Analyses}

\section{Analyses statistiques et traitements mathématiques}

Nous avons analysé l'évolution du volume marchand des arbres résineux et celle du diamètre quadratique moyen des 1000 plus gros arbres résineux à l'hectare pour comprendre les effets de léclaircie sur la production du peuplement et sur le développement des arbres. Nous avons analysé le diamètre quadratique moyen des 1000 plus gros arbres résineux à l'hectare plutôt que celui des arbres marchands, afin détablir une base de comparaison équitable entre les traitements. Les ana- lyses ont été effectuées avec un modèle linéaire mixte à trois facteurs (PROC GLIMMIX SAS 9.3; SAS Institute Inc. 2003). Le traitement et l'année du mesurage après traitement ont été utilisés en effets fixes, les placettes, en effets aléatoires et l'année du mesurage, en mesures répétées. Comme l'intervalle entre les années de mesure était régulier, nous avons utilisé une structure de covariance de type AR(1). Pour tenir compte de la variabilité initiale à l'intérieur du peuplement, nous avons vérifié l'utilité d'inclure la surface terrière initiale, le nombre initial d'arbres, le diamètre quadratique moyen initial ou l'IQS initial comme covariable lors des analyses. La comparaison des valeurs d'AICC (c.-à-d. le critère d'information d’Akaike corrigé pour la taille de léchantillon) a permis de sélectionner la covariable contribuant le mieux à exprimer la variabilité de la sapinière avant traitement (Burnham et Anderson 2002).

Nous avons utilisé l'énoncé LSMESTIMATE de la procédure GLIMMIX pour comparer les valeurs moyennes en fonction du traitement et du temps depuis son application. Pour chaque variable, nous avons comparé les effets de chacune des trois intensités d'éclaircie avec le témoin, en 1997 (immédiatement après traitement) et en 2007, pour un total de 6 comparaisons. Lors de ces comparaisons, les valeurs de probabilité $(p)$ ont été ajustées avec l'option SIMULATE. Nous avons utilisé le seuil de 0,05 pour déterminer les effets significatifs, et vérifié la normalité des résidus ainsi que l'homogénéité de la variance.

Nous avons calculé le volume des arbres avec les équations de Honer et al. (1983) et le volume des arbres marchands avec celle de Perron (2003), à partir d'une hauteur totale prédite au préalable pour chaque arbre. Pour estimer cette hauteur totale, nous avons étalonné une relation hauteur-DHP, par traitement, à partir des données des arbres échantillons (NLMIXED SAS 9.3; SAS Institute Inc. 2003), en utilisant le modèle non linéaire numéro 3 (sans b2) de Bégin et Raulier (1995), avec les placettes en effets aléatoires. Lindice de qualité de station (IQS : hauteur moyenne des 100 plus grosses tiges à l'hectare à 50 ans) a été déterminé à l'aide de l'équation de Pothier et Savard (1998).

\section{Analyses dendrochronologiques}

Pour conserver le patron de croissance radiale relié aux effets locaux (p. ex. : léclaircie), nous avons standardisé les chronologies de largeur des cernes annuels en apposant une ligne horizontale (Cook et Kairiukstis 1990). Cette standardisation a produit des indices de largeur de cernes à partir desquels nous avons généré une chronologie standardisée moyenne pour chaque traitement, en utilisant une moyenne robuste bipondérée (biweighted, Cook et Holmes 1999). Nous avons fait ces analyses à l'aide de la bibliothèque de programmes de dendrochronologie (dplR) du logiciel R (version 2.15.3; Bunn 2008). Pour évaluer l'effet de l'éclaircie, nous avons comparé visuellement la courbe standardisée moyenne des parcelles éclaircies avec celle des parcelles témoins, avant et après traitement. Pour compléter l'analyse visuelle, nous avons calculé un coefficient de corrélation de Pearson pour comparer les courbes entre elles en fonction de l'année durant ces deux périodes. 
Tableau 1. Caractéristiques dendrométriques ( $X$; (erreur type)] pour toutes les espèces d'arbres en fonction de l'intensité d'éclaircie, avant et immédiatement après l'intervention.

\begin{tabular}{|c|c|c|c|c|c|c|c|c|}
\hline & \multicolumn{4}{|c|}{ Avant l'éclaircie (1997) } & \multicolumn{4}{|c|}{ Après l'éclaircie (1997) } \\
\hline & Témoin & $16 \%$ & $25 \%$ & $35 \%$ & Témoin & $16 \%$ & $25 \%$ & $35 \%$ \\
\hline Âge (années) & $56(6)$ & $55(1)$ & $56(6)$ & $54(2)$ & $56(6)$ & $55(1)$ & $56(6)$ & $54(2)$ \\
\hline $\mathrm{H}_{\mathrm{d}}(\mathrm{m})$ & $12,1(1,0)$ & $12,9(0,7)$ & $11,6(0,5)$ & $13,4(0,6)$ & $12,2(1,0)$ & $13,0(0,7)$ & $11,6(0,6)$ & $13,3(0,6)$ \\
\hline $\mathrm{D}_{\mathrm{q}}(\mathrm{cm})$ & $8,4(1,2)$ & $8,2(0,5)$ & $7,9(1,1)$ & $8,5(0,4)$ & $8,6(1,5)$ & $11,5(0,5)$ & $11,4(1,5)$ & $12,8(0,5)$ \\
\hline $\mathrm{D}_{\mathrm{qm}}(\mathrm{cm})$ & $12,0(0,5)$ & $11,7(0,3)$ & $12,1(0,5)$ & $12,3(0,3)$ & $12,0(0,6)$ & $12,1(0,4)$ & $12,5(1,0)$ & $13,2(0,5)$ \\
\hline$N\left(\mathrm{nb} \cdot \mathrm{ha}^{-1}\right)$ & $10958(2891)$ & $10342(960)$ & $11817(2701)$ & $9517\left(\begin{array}{lll}1 & 013\end{array}\right)$ & $10725(3160)$ & $2758(279)$ & $2933(650)$ & $1825(240)$ \\
\hline $\mathrm{N}_{\mathrm{m}}\left(\mathrm{nb} \cdot \mathrm{ha}^{-1}\right)$ & $2842(469)$ & $2875(214)$ & $2333(409)$ & $2883(120)$ & $2925(416)$ & $2292(51)$ & $1733(83)$ & $1658(175)$ \\
\hline $\mathrm{G}\left(\mathrm{m}^{2} \cdot \mathrm{ha}^{-1}\right)$ & $54(2)$ & $54(1)$ & $53(1)$ & $53(1)$ & $53(2)$ & $29(2)$ & $27(<1)$ & $23(1)$ \\
\hline$G_{m}\left(m^{2} \cdot h a^{-1}\right)$ & $33(8)$ & $31(3)$ & $28(7)$ & $34(1)$ & $34(8)$ & $26(2)$ & $21(3)$ & $22(1)$ \\
\hline $\mathrm{V}\left(\mathrm{m}^{3} \cdot \mathrm{ha}^{-1}\right)$ & $257(24)$ & $235(11)$ & $247(19)$ & $234(10)$ & $255(24)$ & $142(9)$ & $136(7)$ & $118(3)$ \\
\hline$V_{m}\left(m^{3} \cdot h a^{-1}\right)$ & $122(40)$ & $107(15)$ & $102(35)$ & $125(9)$ & $125(44)$ & $94(11)$ & $78(20)$ & $89(5)$ \\
\hline
\end{tabular}

$\mathrm{H}_{\mathrm{d}}$ : hauteur modélisée des 100 plus gros arbres; $\mathrm{D}_{\mathrm{q}}$ : diamètre quadratique moyen des arbres (diamètre à hauteur de poitrine $\left.(\mathrm{DHP}) \geq 1,1 \mathrm{~cm}\right) ; \mathrm{D}_{\mathrm{qm}}:$ diamètre quadratique moyen des arbres marchands (DHP $\geq 9,1 \mathrm{~cm}$ ); $\mathrm{N}$ : nombre d’arbres; $\mathrm{N}_{\mathrm{m}}$ : nombre d’arbres marchands; $\mathrm{G}$ : surface terrière des arbres; $\mathrm{G}_{\mathrm{m}}$ : surface terrière des arbres marchands; $\mathrm{V}$ : volume des arbres; $\mathrm{V}_{\mathrm{m}}$ : volume des arbres marchands.

\begin{abstract}
Résultats
Caractéristiques du peuplement avant et immédiatement après l'éclaircie

Avant l'éclaircie, la sapinière était âgée de 55 ans et avait un volume total moyen de $243 \mathrm{~m}^{3} \cdot \mathrm{ha}^{-1}$ et un volume marchand de $114 \mathrm{~m}^{3} \cdot \mathrm{ha}^{-1}$ (Tableau 1). Elle était constituée, en moyenne,

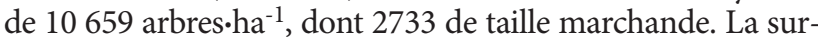
face terrière totale moyenne était de $54 \mathrm{~m}^{2} \cdot \mathrm{ha}^{-1}$ alors que celle marchande était de $32 \mathrm{~m}^{2} \cdot \mathrm{ha}^{-1}$. Le diamètre quadratique moyen était de $8,30 \mathrm{~cm}$ pour tous les arbres et de $12,03 \mathrm{~cm}$ pour les arbres marchands. Les caractéristiques des arbres échantillons étaient semblables entre les traitements. Ils avaient un facteur délancement (c.-à-d. leur rapport hauteur/DHP) de $80(p=0,627)$, un pourcentage de houppier vivant de $54 \%(p=0,056)$, un diamètre moyen de $14,88 \mathrm{~cm}$ $(p=0,834)$ et une hauteur moyenne de $11,4 \mathrm{~m}(p=0,614)$, conférant à la sapinière un IQS de $11 \mathrm{~m}(p=0,379)$. Les trois intensités déclaircie réalisées correspondent respectivement à $40 \%, 45 \%$ et $50 \%$ du volume total, à $12 \%, 24 \%$ et $29 \%$ du volume marchand, à $73 \%, 75 \%$ et 81
\end{abstract} $\%$ du nombre total d'arbres ou à $20 \%$, $26 \%$ et $42 \%$ du nombre d'arbres marchands, à $46 \%, 49 \%$ et $57 \%$ de la surface terrière totale ou à $16 \%, 25 \%$ et $35 \%$ de la surface terrière marchande (Tableau 1). Les sapins baumiers représentaient plus de $80 \%$ des arbres avant et après léclaircie. Les espèces compagnes étaient le bouleau à papier, l'épinette blanche (Picea glauca (Moench) Voss) et l'épinette noire (Picea mariana (Mill.) B.S.P.).

\section{Évolution de la production}

Le volume marchand résineux a augmenté dans le temps en interaction avec l'intensité de léclaircie (Tableau 2). Ce volume a été positivement influencé par la surface terrière marchande avant léclaircie (c.-à-d. la covariable; Tableau 1; Tableau 2). Immédiatement après le traitement, le volume marchand résineux dans les placettes fortement éclaircies était 1,5 fois plus faible que dans les placettes témoins (Fig. 3a; $p=0,047$ ). Par contre, 10 ans plus tard, le volume était semblable dans ces deux traitements $(p=0,627)$, car l'augmentation a été de $74 \mathrm{~m}^{3} \cdot \mathrm{ha}^{-1}$ dans les placettes fortement éclaircies, mais de seulement $51 \mathrm{~m}^{3} \cdot \mathrm{ha}^{-1}$ dans celles témoins. Les différences de volume nétaient pas significatives entre les placettes faiblement ou modérément éclaircies et les placettes témoins, aussi bien après traitement $(p \geq 0,343)$ que 10 ans plus tard $(p \geq 0,991)$. Les gains en production dans les placettes fortement éclaircies sont attribuables essentiellement à la croissance des arbres qui avaient déjà une taille marchande immédiatement après léclaircie. En effet, la contribution des nouveaux arbres marchands depuis 1997 ne représentait que $2 \%$ du volume marchand en 2007 (Fig. 3b)

Tableau 2. Résultats des analyses de covariance (nombre de degrés de liberté au numérateur (dll) et probabilités $(\mathrm{Pr}>\mathrm{F})$ ) du volume des arbres sur pied de taille marchande (diamètre à hauteur de poitrine $\geq 9,1 \mathrm{~cm}$ ) résineux et du diamètre quadratique moyen des 1000 plus gros arbres résineux à l'hectare (Dq1000).

\begin{tabular}{|c|c|c|c|c|c|c|c|c|}
\hline & \multicolumn{2}{|c|}{$\begin{array}{l}\text { Intensité } \\
\text { d'éclaircie }\end{array}$} & \multicolumn{2}{|c|}{ Année } & \multicolumn{2}{|c|}{$\begin{array}{l}\text { Intensité } \\
\text { d'éclaircie } \\
\text { x Année }\end{array}$} & \multicolumn{2}{|c|}{ Covariable } \\
\hline & dl & $\operatorname{Pr}>F$ & dl & $\operatorname{Pr}>F$ & dl & $\operatorname{Pr}>F$ & dl & $\operatorname{Pr}>F$ \\
\hline Volume marchand & 3 & 0,278 & 2 & $<0,001$ & 6 & 0,009 & 1 & $<0,001^{*}$ \\
\hline Dq1000 & 3 & 0,203 & 2 & $<0,001$ & 6 & $<0,001$ & 1 & $<0,001^{\dagger}$ \\
\hline
\end{tabular}

* Surface terrière des arbres marchands résineux avant traitement.

$†$ Diamètre quadratique moyen des arbres marchands résineux avant traitement. Les degrés de liberté au dénominateur, certaines relations non significatives et les effets aléatoires ne sont pas présentés pour alléger la présentation. Les valeurs significatives sont en gras. 

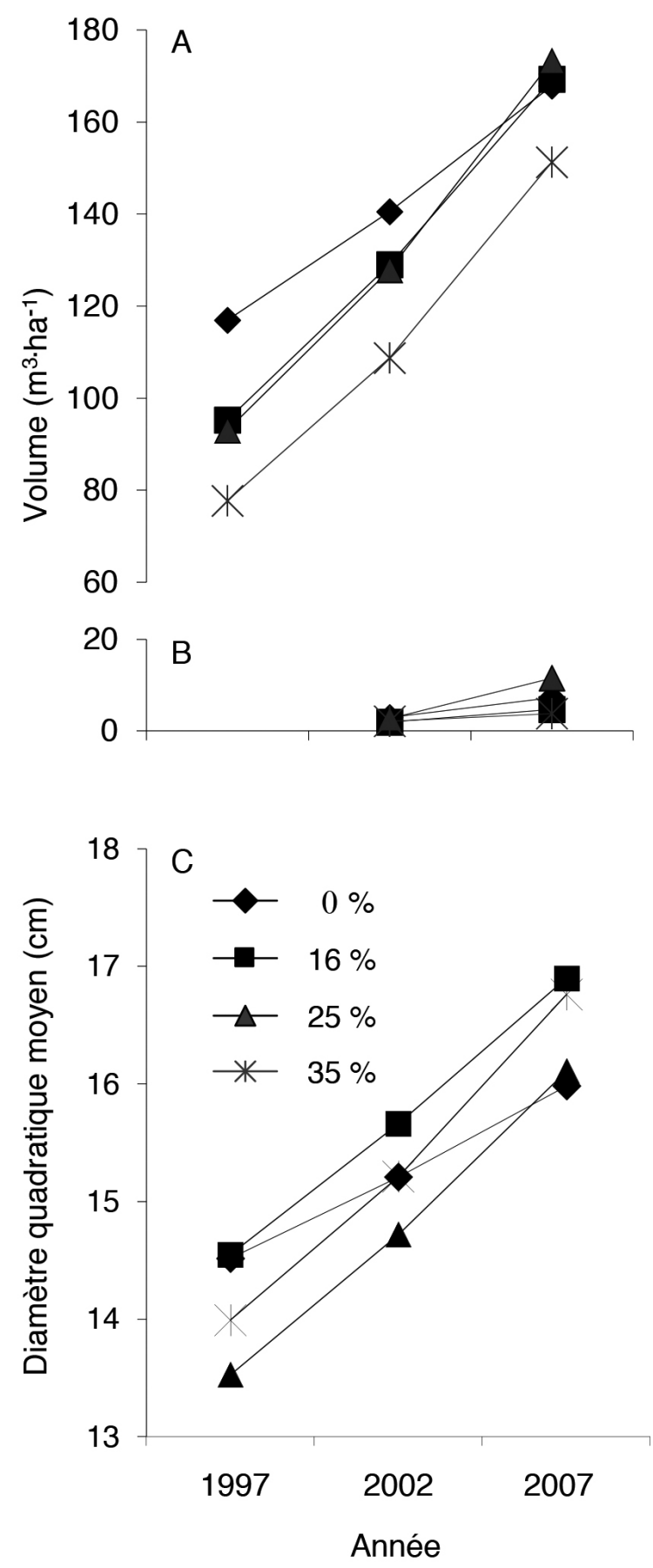

Fig. 3. Évolution dans le temps, après traitement, de la valeur estimée $(\bar{X})$ du volume (erreur type $=10 \mathrm{~m}^{3} \cdot \mathrm{ha}^{-1}$ ) des arbres marchands (diamètre à hauteur de poitrine $\geq 9,1 \mathrm{~cm}$ ) résineux (A : arbres marchands en 1997 et nouveaux arbres marchands depuis 1997; B : nouveaux arbres marchands depuis 1997) et du diamètre quadratique moyen (erreur type $=0,27 \mathrm{~cm}$ ) des 1000 plus gros arbres résineux à l'hectare (C), par intensité d'éclaircie de la surface terrière des arbres marchands.
Le diamètre quadratique moyen des 1000 plus gros arbres résineux à l'hectare a augmenté dans le temps, en interaction avec l'intensité de l'éclaircie (Tableau 2). Laugmentation a également été fonction du diamètre quadratique moyen des arbres résineux de taille marchande avant léclaircie (c.-à-d. la covariable; Tableau 1; Tableau 2). Ce diamètre a augmenté plus rapidement dans les placettes éclaircies que dans celles témoins ( $p=0,003$; Fig. 3c). Notamment, il a augmenté deux fois plus rapidement dans les placettes fortement éclaircies $(p<0,001)$. En définitive, 10 ans après traitement, les placettes fortement éclaircies avaient retrouvé un volume marchand semblable à celui des placettes témoins, mais ce volume était distribué sur $43 \%$ moins d’arbres, et le diamètre quadratique moyen a crû deux fois plus rapidement chez les 1000 plus gros d’entre eux.

Le ralentissement de croissance et l'effet positif de léclaircie de forte intensité étaient visibles dans les courbes dendrochronologiques (Fig. 4). La courbe des placettes fortement éclaircies et celle des placettes témoins descendent généralement jusqu’au moment de léclaircie (1947-1997; coefficient de Pearson : $r=0,72, p<0,001)$. Toutefois, après le traitement, la courbe des placettes fortement éclaircies monte, alors que celle des placettes témoins continue de descendre $(r=-$ $0,55, p=0,078)$. L'effet de léclaircie semble se stabiliser après neuf ans. À partir de 2006, la courbe des placettes fortement éclaircies cesse de monter et devient parallèle à celle des placettes témoins, mais à une valeur plus de deux fois plus élevée.

Enfin, 10 ans après léclaircie, le pourcentage de houppier vivant des arbres échantillons était de $39 \%$ dans les placettes témoins, alors qu'il atteignait $51 \%$ dans les placettes fortement éclaircies. De plus, la mortalité des arbres marchands était de $11 \%$ dans les placettes témoins, mais de seulement $2 \%$ dans les placettes éclaircies.

\section{Discussion}

Évolution de la production

En intégrant la dendrochronologie à la dendrométrie, nous avons pu démontrer que léclaircie par le bas de forte intensité a remplacé l'autoéclaircie et a rétabli la croissance des arbres dans la sapinière très dense, augmentant ainsi sa productivité. Dix ans après traitement, les placettes fortement éclaircies avaient retrouvé un volume marchand semblable à celui des placettes non éclaircies, mais celui-ci était distribué sur moins d’arbres, et le diamètre quadratique moyen a crû deux fois plus rapidement chez les 1000 plus gros dentre eux. Ainsi, conformément à notre hypothèse, la courbe de production des placettes fortement éclaircies a convergé vers la courbe des placettes non éclaircie (Fig. 1; McKinnon et al. 2006). L’obtention de courbes post-éclaircie convergentes a rarement été rapportée dans la littérature. Léclaircie précommerciale ou commerciale améliore le diamètre des arbres, mais pas forcément le volume marchand du peuplement (CCSMAF 2002, 2003; Raulier et al. 2003, McKinnon et al. 2006). On s'attend habituellement à ce que les courbes de production des peuplements éclaircis et non éclaircis soient parallèles après léclaircie commerciale, et convergentes après l'éclaircie précommerciale (CCSMAF 2002, 2003). Néanmoins, certains auteurs suggèrent qu'il est possible d’obtenir un faible gain en volume marchand sur les stations les plus productives, à condition de multiplier les éclaircies commerciales de faible intensité et d'allonger la révolution (McKinnon et al. 2006, Pelletier et 


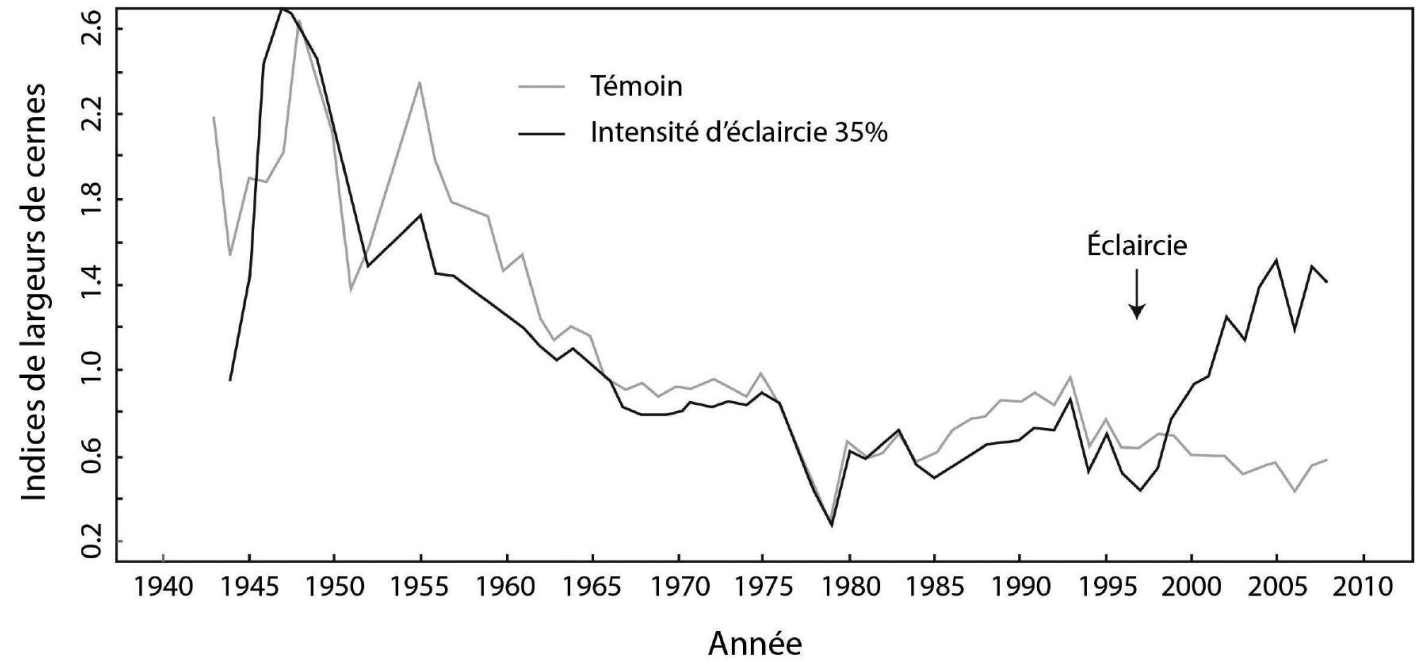

Fig. 4. Chronologies moyennes des indices de largeur des cernes annuels de 18 sapins baumiers provenant des placettes éclaircies à $35 \%$ et de 22 sapins baumiers provenant des placettes témoins.

Pitt 2008). Dans létude actuelle, la courbe de production convergente a plutôt été obtenue sur une station apparemment peu productive, peu après une seule éclaircie intermédiaire de forte intensité. Ce résultat est d’autant plus surprenant que la forte densité de la sapinière avait perduré suffisamment longtemps pour que la compétition ralentisse la croissance des arbres. En effet, l'éclaircie est davantage reconnue pour prévenir que pour rétablir un retard de croissance causé par la compétition reliée à la densité (Smith et al. 1997, McCarthy et Weetman 2007a). Toutefois, lanalyse dendrochronologique nous a permis de confirmer quaprès un ralentissement prolongé de la croissance des arbres, léclaircie a engendré une reprise rapide de la croissance radiale, qui ne s'est stabilisée qu'une dizaine d'années plus tard. Cette stabilisation a possiblement été causée par laugmentation de la compétition, à la suite de lexpansion du houppier des arbres. Léclaircie avait néanmoins toujours un effet positif sur la croissance, puisque celle-ci sest stabilisée à des valeurs plus de deux fois plus grandes dans les placettes éclaircies que dans les placettes témoins.

La réaction convergente du volume marchand de la sapinière fortement éclaircie pourrait s'expliquer en partie par l'immaturité physiologique des arbres. Lâge physiologique d'un arbre (c.-à-d. lâge associé au stade de développement) est dépendant de son âge chronologique (c.-à-d. le nombre d'années depuis la germination), mais aussi des ressources disponibles (Blais 1958, Greenwood 1995, Millet 2012). Ainsi, les sapins qui subissent une compétition pour les ressources pourraient ne pas encore avoir atteint leur maturité physiologique, malgré un âge chronologique avancé. Ces sapins baumiers pourraient donc avoir maintenu des caractéristiques morpho-physiologiques d'arbres plus jeunes leur permettant de bénéficier d'une ouverture tardive du couvert. Par exemple, dans la présente étude, la proportion de houppier vivant des sapins baumiers était toujours largement supérieure au seuil minimal défini pour la réalisation d'une éclair- cie (c.-à-d. $33 \%$ de houppier vivant : CCSMAF 2003). De plus, nous avons observé peu de mortalité dans la sapinière témoin, et très peu dans la sapinière éclaircie, malgré le fait que les sapins approchaient de lâge chronologique auquel ils sont habituellement cariés (McCarthy et Weetman 2007b).

La réaction convergente du volume marchand de la sapinière éclaircie pourrait aussi s'expliquer par le réchauffement du sol de la station après laugmentation de la lumière. Dans les zones boréales (Saucier et al. 2009), le réchauffement du sol peut accélérer la minéralisation (Thibodeau et al. 2000), allonger la période d'activité racinaire et augmenter la durée de la saison de croissance (Piene 1978, Puhe 2003). Aussi, la station était possiblement plus productive que ne l'indiquait I'IQS. En effet, selon Ker (1976), un IQS d'environ $16 \mathrm{~m}$ et non $11 \mathrm{~m}$ devrait être nécessaire pour qu’une sapinière produise un volume total similaire à celui de la sapinière de notre étude avant traitement. La sous-estimation de l'IQS pourrait s'expliquer par un ralentissement de la croissance en hauteur des arbres due à la densité. Enfin, bien que la relation hauteurDHP utilisée dans cette étude soit reconnue dans la littérature comme valide pour calculer le volume, les valeurs obtenues sont dépendantes de cette relation.

Des arrosages aériens d'insecticides ont été réalisés en 1976 et en 1984 dans la région du dispositif, pour protéger les sapinières contre lépidémie de TBE de 1978 (Leblanc et Bélanger 2000, Boulanger et Arseneault 2004). En conséquence, la sapinière étudiée n’a subi qu'une légère défoliation et une faible mortalité (c.-à-d. $<25 \%$ : MRNF 2011). Or, les épidémies de TBE peuvent contribuer au processus d'autoéclaircie en affaiblissant des arbres qui seront plus susceptibles de mourir de la compétition reliée à la densité (Baskerville 1975, McCarthy et Weetman 2007b, MacLean et Andersen 2008). Les arrosages pourraient donc avoir contribué au dysfonctionnement de lautoéclaircie et au ralentissement de croissance dans les sapinières très denses, en atténuant la contribution de lépidémie de 1978 à ce processus écologique. 
Implications pour l'aménagement de sapinières très denses En définitive, tant que les sapins baumiers nont pas atteint leur maturité physiologique, l'éclaircie par le bas peut rétablir la production de matière ligneuse dans des sapinières très denses ayant atteint l'âge de la maturité commerciale. En aussi peu que 10 ans, de telles sapinières éclaircies peuvent produire un volume marchand semblable à celui de sapinières non traitées. Ce volume se retrouve alors distribué sur un plus petit nombre d'arbres, qui acquièrent des dimensions commerciales intéressantes plus rapidement. De tels arbres peuvent aussi être moins coûteux à récolter et à transformer (Gingras et Favreau 2005, Karlsson 2006). Léclaircie ayant toujours un effet positif sur la croissance après 10 ans, il sera intéressant d'évaluer l'opportunité de réaliser une seconde éclaircie avant la coupe finale. Toutefois, avant toute intervention, il sera important de confirmer que les sapinières très denses n'ont pas encore atteint leur maturité physiologique, afin de s'assurer que les éclaircies auront le potentiel de stimuler la production. Une bonne indication de l'immaturité physiologique de sapinières très denses est l'inadéquation entre l'atteinte de l'âge de maturité commerciale et les caractéristiques dendrométriques de sapinières matures au développement normal (Ker 1976; Pothier et Savard 1998; Raulier et al. 2003). Enfin, avant de considérer l'application d'éclaircies de ce type à une échelle opérationnelle, il sera important d'évaluer l'effet de sentiers de débardage sur la production et sur les risques de chablis.

\section{Remerciements}

Nous tenons à remercier David Pothier et Denis Villeneuve pour la conception du dispositif expérimental ainsi qu’Alain Langlois, Louis Faucher, Richard Verret et Simon Pouliot pour le travail technique lors de l'installation du dispositif, les trois mesurages et l'analyse en laboratoire. Nous voulons aussi remercier Geneviève Picher et Isabelle Auger pour leur aide lors des analyses statistiques, et Denise Tousignant pour la révision linguistique. Enfin, ce manuscrit a bénéficié des commentaires constructifs de deux réviseurs anonymes.

\section{Références}

Barrette, M., L. Bélanger and L. De Grandpré. 2010. Preindustrial reconstruction of a perhumid midboreal landscape, Anticosti Island, Quebec. Can. J. For. Res. 2010, 40(5): 928-942. doi:10.1139/X10-040. Baskerville, G. 1975. Spruce budworm - super silviculturist. For. Chron. $51: 4-6$.

Bégin, J. and F. Raulier. 1995. Comparison of different approaches, models and sample sizes for determining local height-diameter relations. Can. J. For. Res. 25(8) : 1303-1312.

Blais, J.R. 1958. The vulnerability of balsam fir to spruce budworm attack in northwestern Ontario, with special reference to the physiological age of the tree. For. Chron. 34(4) : 405-422.

Boulanger, Y. and D. Arseneault. 2004. Spruce budworm outbreaks in eastern Quebec over the last 450 years. Can. J. For. Res. 34(5) : 1035-1043

Bunn, A.G. 2008. A dendrochronology program library in R (dplR). Dendrochronologia. 26(2) : 115-124. doi:10.1016/j.dendro.2008.01.002.

Burnham, K.P. and D.R. Anderson. 2002. Model selection and multimodel inference, a practical information-theoritic approach, second edition. New York : Springer $487 \mathrm{p}$

CCSMAF (Comité consultatif scientifique du Manuel d'aménagement forestier). 2002. Le traitement déclaircie précommerciale pour le groupe prioritaire SEPM. Avis scientifique. Ministère des Ressources naturelles, Direction de la recherche forestière. 126 p.
CCSMAF (Comité consultatif scientifique du Manuel d'aménagement forestier). 2003. Éclaircie commerciale pour le groupe prioritaire SEPM. Avis scientifique. Gouvernement du Québec, Forêt Québec, Ministère des Ressources naturelles, Direction de la recherche forestière. $80 \mathrm{p}$.

Cook, E.R. and R.L. Holmes. 1999. Program ARSTAN-Chronology development with statistical analysis (Users manual for program ARSTAN). Laboratory of Tree-Ring Research, University of Arizona, Tucson, AZ, USA. 9 p.

Cook, E.R. and L.A. Kairiukstis. 1990. Methods of dendrochronology applications in the environmental sciences. Kluwer Academic Publishers, Dordrecht, Pays-Bas.

Côté, S. and L. Bélanger. 1991. Variations de la régénération préétablie dans les sapinières boréales en fonction de leurs caractéristiques écologiques. Can. J. For. Res. 21(12) : 1779-1795.

Environnement Canada. 1993. Normales climatiques au Canada 1961-90. 157 p.

Franklin, J.F., R.J. Mitchell and B.J. Palik. 2007. Natural Disturbance and Stand Development Principles for Ecological Forestry, USDA Forest Service General Tech. Rpt. NRS-19 (Northern Research Station, Newton Square, PA).

Greenwood, M.S. 1995. Juvenility and maturation in conifers: current concepts. Tree Physiol. 15 (7-8) : 433-438.

Gingras, J.-F. and J. Favreau. 2005. Effet de la longueur des billes et du nombre de produits sur la productivité du procédé par bois tronçonnés en forêt boréale. FPInnovations. Advantage 6(10) : 1-8.

Holmes, R.L. 1983. Computer-assisted quality control in tree-ring dating and measurement. Tree-Ring Bull. 43 : 69-78.

Honer, T.G., M.F. Ker and I.S. Alemdag. 1983. Metric timber tables for the commercial tree species of central and eastern Canada. Information Report M-X-140. Canadian Forest Service, Maritimes Forest Research Center. 23 p.

Karlsson, K. 2006. Impact of the thinning regime on the mean diameter of the largest stems by diameter at breast height in even-aged Picea abies stands. Scand. J. For. Res. 21(1) : 20-31.

Ker, M.F. 1976. Metric yield tables for major forest cover types of Newfoudland. Can. For. Serv. Newfoundland For. Res. Cent. Inf. Rep. $\mathrm{n}^{\circ} \mathrm{N}-\mathrm{X}-141$.

Leblanc, M. and L. Bélanger. 2000. La sapinière vierge de la forêt Montmorency et de sa région : une forêt boréale distincte. Ministère des Ressources naturelles du Québec. Mémoire de recherche forestière no 136. Québec, QC. 91 p.

MacLean, D.A. and A.R. Andersen. 2008. Impact of a spruce budworm outbreak in balsam fir and subsequent stand development over a 40-year period. For. Chron. 84(1) : 60-69.

McCarthy, J.W. and G. Weetman. 2007a. Self-thinning dynamics in a balsam fir (Abies balsamea (L.) Mill.) insect-mediated boreal forest chronosequence. For. Ecol. Manage. 241(1-3) : 295-309. doi:10.1016/ j.foreco.2006.12.030.

McCarthy, J.W. and G. Weetman. 2007b. Stand structure and development of an insect-mediated boreal forest landscape. For. Ecol. Manage. 241(1-3) : 101-114. doi:10.1016/j.foreco.2006.12.030.

McKinnon, L.M., G.J. Kayahara and R.G. White. 2006. Biological framework for commercial thinning even-aged single-species stands of jack pine, white spruce, and black spruce in Ontario. Ontario Ministry of Natural Resources, Science and Information Branch, Northeast Science and Information Section, South Porcupine, ON. Technical Report TR-046. 126 p.

Millet, J. 2012. L'architecture des arbres des régions tempérées : son histoire, ses concepts, ses usages. Éditions MultiMondes. 411 p.

Morin, H. and D. Laprise. 1997. Seeding bank dynamics in boreal balsam fir forests. Can. J. For. Res. 27(9) : 1442-1451.

MRNF. 2011. Aires infestées par la tordeuse des bourgeons de l'épinette, aires traitées par arrosages et mortalité due à la TBE, au Québec, entre 1966 et 1992. Ministère des Ressources naturelles et de la Faune. Direction de la protection des forêts. Couches numériques consultées en 2011. 
Nyland, R.D. 2007. Silviculture: concepts and applications. Second edition. Waveland Press, inc. Long Grove, Il, USA. 682 p.

Pelletier, G. and D.G. Pitt. 2008. Silvicultural responses of two spruce plantations to midrotation commercial thinning in New Brunswick. Can. J. For. Res. 38(4) : 851-867. doi : 10.1139/X07-173. Perron, J.-Y. 2003. Tarif de cubage général, volume marchand brut. $3^{\mathrm{e}}$ publication. Ministère des Ressources naturelles de la faune et des Parcs, Forêt Québec, Direction des inventaires forestiers. 53 p.

Piene, H. 1978. Effects of increasing spacing on carbon mineralization rates and temperature in a stand of young balsam fir. Can. J. For. Res. 8(4) : 398-406.

Pothier, D. and F. Savard. 1998. Actualisation des tables de production pour les principales espèces forestières du Québec. Gouvernement du Québec, ministère des Ressources naturelles et de la Faune, Direction des inventaires forestiers. Publication RN98-3054. 184 p. Puhe, J. 2003. Growth and development of the root system of Norway spruce (Picea abies) in forest stands-a review. For. Ecol. Manage. 175(1-3) : 253-273.

Raulier, F., D. Pothier and P.Y. Bernier. 2003. Predicting the effect of thinning on growth of dense balsam fir stands using a processbased tree growth model. Canadian Journal of Forest Research, $33(3)$ : 509-520.
SAS Institute Inc. 2003. The SAS system for Windows, version 9.3. SAS Institute Inc. Cary, NC, USA.

Saucier, J.-P., P. Grondin, A. Robitaille, J. Gosselin, C. Morneau, P.J.H. Richard, J. Brisson, L. Sirois, A. Leduc, H. Morin, É. Thiffault, S. Gauthier, C. Lavoie and S. Payette. 2009. Écologie forestière. Dans Ordre des ingénieurs forestiers du Québec, Manuel de foresterie, $2^{\mathrm{e}}$ éd. Ouvrage collectif, Éditions MultiMondes, Québec, p. 164-316.

Smith, D.M., B.C. Larson, M.J. Kelty and P.M.S. Asthon. 1997. The practice of sylviculture: applied forest Ecology, John Wiley \& Sons, New York, $537 \mathrm{p}$.

Thibodeau, L., R. Raymond, C. Camire and A.D. Munson. 2000. Impact of precommercial thinning in balsam fir stands on soil nitrogen dynamics, microbial biomass, decomposition, and foliar nutrition. Can. J. For. Res. 30(2) : 229-238. 